\title{
Interlocking Assembled 3D Auxetic Cellular Structures
}

\author{
Xin-Tao Wang, Xiao-Wen Li, Li Ma* \\ Center for Composite Materials, Harbin Institute of Technology, Harbin 150080, PR China
}

\section{Abstract}

As promising metamaterials, 3D periodic auxetic cellular structures (PACSs) have attracted great interest. However, they usually consist of intricate geometries which make their fabrication a significant challenge. The present paper is focused on introducing the interlocking assembly concept into the fabrication of 3D PACSs. There are distinct advantages of the interlocking assembly method compared with the additive manufacturing methods mainly used before. Based on the interlocking assembly method, the dependences of mechanical properties mainly including the Poisson's ratio and the Young's modulus of the structure on the re-entrant angle were investigated through a combination of uniaxial compression experiments and numerical simulations, excellent qualitative and quantitative agreement was found. Using the experimentally verified numerical model, the effects of the strut thickness and the ratio of the vertical strut length to oblique strut length on the mechanical properties of the structure were investigated. Results show that the compression modulus of the structure will increase with the structure becomes more re-entrant, but there exists an extreme value for Poisson's ratio with the re-entrant angle around $45^{\circ}$ which differs from former studies. With the thickening of the struts the compression modulus of the structure monotonously increases and the Poisson's ratio of the structure will gradually changes from negative to positive then gradually approaches to the Poisson's ratio of the parent material. The vertical strut length to oblique strut length ratio plays fewer roles on the mechanical properties

\footnotetext{
${ }^{*}$ Corresponding author, Tel.:+86 451 86402376; fax: +86 45186402376.

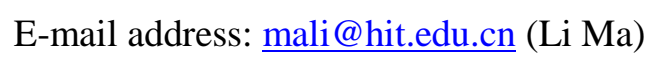


compared with the re-entrant angle and the strut thickness.

\section{Keywords: Auxetic; Cellular materials; Mechanical properties; Interlocking assembled}

\section{method}

\section{Introduction}

Auxetic means that the material or structure possesses negative Poisson's ratio. That is, they will expand in lateral direction when stretched and contract when compressed, which is contrary to common materials and structures. Actually, many auxetic materials have been discovered long before in nature [1-5]. But extensive research on auxetic materials started from the seminal work done by Lakes, in which a three-step manufacturing method of auxetic foams from conventional open-cell foam was introduced [6]. Since Lakes' work, extensive research on auxetic materials have been done because of the fascinating properties introduced by the negative Poisson's ratio effect, such as increased shear modulus [7], indentation resistance [8, 9], fracture toughness [6, 10], energy absorption [11], porosity/permeability variation with strain [12, 13], synclastic curvature [14]. Due to these excellent properties, auxetic materials have great potential for many applications fields such as aerospace, protection, biomedical, sensors and so on [15]. The state of art on the auxetic material and structures can be found in a recent monograph by Lim [16].

In recent years, 3D periodic auxetic cellular structures (PACSs) attracted considerable interest and became a focus of auxetic material research [17-26]. From these researches, it can be found that nearly all 3D PACSs were manufactured by additive manufacturing methods due to their complicated structure. There are a lot of advantages of additive manufacturing, such as its ability to produce structures of arbitrary geometry in a well-defined manner, decreased lead time from concept to product, ease of design and process modification and so on[18, 25]. Though there are also limitations for it. Additive manufacturing method will introduce defects to the structure such as 
rough surface [18], take Electron Beam Melting (EBM) process for example, as shown in the Figure 1a, irregular cross sections [18] as shown in the Figure 1c, and defects caused by stair stepping effect [27] as shown in the Figure 1d. Additionally, mass production and cost are also problems for additive manufacturing method, and the materials can be used as well as the scale of the structures are also limited by the additive manufacturing equipment. Till now, fabrication of fiber reinforced plastic composite structure is still a challenge for additive manufacturing method. So, in the development of complicated 3D auxetic structures both additive manufacturing and traditional manufacturing methods are need.

In this paper, a novel manufacturing method which adopts the interlocking assembly concept is proposed. Interlocking mechanism has been used in the analysis of a $2 \mathrm{D}$ auxetic model by Ravirala et al. [28], and here it is introduced into the fabrication of 3D re-entrant auxetic structure. The proposed method can overcome the defects of additive manufacturing method mentioned above, and it will greatly reduce the manufacturing expenses. In addition, this novel method is suitable for any kind of material that can be adhesively bonded (e.g. plastic and fiber reinforce composite) or welded (e.g. metal). Based on the proposed method, a series of test specimens were manufactured. The auxetic responses of these structures were investigated through a combination of uniaxial compression experiments and numerical simulations, and excellent qualitative and quantitative agreements were found. With the experimentally verified numerical model, the effects of the strut thickness and the vertical strut length to oblique strut length ratio on the mechanical properties were simulated in turn. Then the roles these main design parameters play on the structure's mechanical properties were analyzed.

\section{Structure and design parameters}


The 3D auxetic structure studied in this work is extended from the $2 \mathrm{D}$ re-entrant hexagonal honeycomb structure. Many researchers have theoretically studied the mechanical properties of the 2D re-entrant hexagonal honeycomb structure [29-32], though it has been shown that the 2D approximations fail to model the properties of the 3D structure accurately [30]. There are also few theoretical studies analyzing the mechanical properties of the 3D structures; however their approximations are too simple that it may be unrealistic in the real system [33], or they got similar results to the 2D model [18]. There also lack of systemic numerical investigation and experimental research on the mechanical properties of the 3D structure. The $2 \mathrm{D}$ and $3 \mathrm{D}$ structures are shown in Figure 2. Both the $2 \mathrm{D}$ and $3 \mathrm{D}$ structures are consisting of vertical struts and oblique struts in a re-entrant manner. The 3D structure can be taken as being consist of many umbrella shaped elements (as the two shown in Figure $\mathbf{2 b}$ ) in a specific manner, similar to the 3D auxetic structure proposed by Schwerdtfeger [25], the mechanism responsible for the auxeticity can be taken as the folding and unfolding of the umbrella shaped elements. The unit cell of the 3D structure is shown in Figure 2c. Considering the symmetry of the 3D structure (seen in Figure $\mathbf{2 b}$ and Figure 2c), the direction $Y$, direction $X_{1}$ and direction $X_{2}$ are assigned. In this study, the mechanical properties of the structure under the load applied in direction $Y$ are of concern, so direction $X_{1}$ and direction $\mathrm{X}_{2}$ were defined as the lateral directions.

For the unit cell of 2D re-entrant auxetic structure, the in-plane geometry can be described in terms of five parameters as shown in Figure 2d: length of the vertical struts $(H)$, length of the oblique struts $(L)$, the re-entrant angle $(\theta)$, width of the vertical struts $\left(t_{1}\right)$ and width of the oblique struts $\left(t_{2}\right)$. For the 3D re-entrant auxetic structure, there need to be two more parameters which are the depth of the vertical strut $\left(t_{3}\right)$ and oblique strut $\left(t_{4}\right)$, as shown in Figure 2e. In this study it is 
supposed that the struts both vertical and oblique have the same square cross section, so it can be assumed that $t_{1}=t_{2}=t_{3}=t_{4}=t$.

To ensure the physically realization of the structure, the design parameters should satisfy geometric compatibility constrains. Formerly, most researchers neglected the effect of the strut cross section dimension on the geometric compatibility constrains. For example, to ensure that the tips of the structure do not overlap, it was stated that the following inequation must be satisfied $[34,35]$.

$$
2 L \cos \theta<H
$$

If the effect of $t$ is taken into consideration, the constrain condition will turn into

$$
\frac{(1-\cos \theta) t}{\sin \theta}+2 L \cos \theta<H
$$

To avoid the overlap of adjacent vertical struts which share a common oblique strut, the following inequation must be satisfied.

$$
t<L \sin \theta
$$

The relative density of lattice truss is the ratio of the unit density to the solid from which it is made, or equivalently the volume fraction of truss members occupying the unit cell [36]. As it was assumed that $t_{1}=t_{2}=t_{3}=t_{4}=t$, the relative density $\rho_{r}$ of the $3 \mathrm{D}$ re-entrant structure can be deduced from the geometry of its unit cell (as shown in Figure 2c), and it could be expressed by the design parameters as

$$
\rho_{r}=\frac{4 t^{2}\left[H+\frac{(1-\cos \theta)}{\sin \theta} t\right]+16 t^{2}\left(L-\frac{t}{\sin \theta}\right)}{2(H-L \cos \theta) \cdot(2 L \sin \theta)^{2}}=\frac{\left[\alpha+4-\frac{(3+\cos \theta)}{\sin \theta} \frac{t}{L}\right] t^{2}}{2(\alpha-\cos \theta) \cdot(L \sin \theta)^{2}}
$$

where $\alpha=H / L$ is the ratio of the vertical strut length to oblique strut length. The denominator in Equation (4) calculates the volume of the unit cell; the first term of the numerator calculates the 
volume of the vertical struts in the unit cell, and the part added to $H$ represents the elongation of the vertical strut due to the thickness $t$; the second term of the numerator calculates the volume of the oblique struts in the unit cell, and the part subtracted from $L$ represents the reduction of the oblique struts due to the overlap of the vertical struts and oblique struts.

\section{The interlocking assembly method}

In the interlocking process, the $3 \mathrm{D}$ auxetic structure was decomposed into four types of $2 \mathrm{D}$ re-entrant component parts, and they were indicated as Part-1, Part-2, Part-3 and Part-4, as shown in Figure 3b. It can be seen that Part-3 and Part-4 are half of Part-1 and Part- 2 respectively. All the component parts were slightly modified by adding small blocks around the interlocking slots at both ends (the small blocks are in dark color in Figure 3c) to facilitate the interlocking of the outmost component parts. As they are all simple 2D structures, it is easy to manufacture them with conventional machining methods such as stamping, cutting, cast and so on. Take Part-1 and Part-2 for example, they can be cut from a panel sheet, as shown in Figure 4. And there are well-developed methods with high accuracy and low surface roughness of cutting panel sheet, such as computer numerical control machine, laser beam cutting, electro-discharge cutting and so on. The upper and lower surface of the parts could be assured to be smooth if the panel sheet has smooth surfaces, and panel sheet with smooth surfaces can be easily obtained. So the high surface smoothness and the regular square cross section of the struts both vertical and oblique could be assured. The surface image of an oblique strut manufactured by wire-electrode cutting with the same measuring scale in Figure 1a is shown in Figure 1b, and the surface qualities can be easily evaluated by comparing these two images.

The interlocking process is schematically described in Figure 3c, Part-3 and Part-4 are set as the basis of the structure, and they were set parallel and alternately, and the distance between the prior 
component part and the next component part is determined by the horizontal distance between adjacent interlocking slots. After the basis being set, Part-1 and Part- 2 were used to carry on the construction, and they are also set parallel and alternately, as shown in Figure 3c, the interlocking of the component parts is indicated by the arrows. After one layer being interlocked, the next layer can be interlocked on the basis of the completed layer with the same method. When the structure has been constructed to the number of layers (number of unit cell in the height direction) needed, Part-3 and Part-4 need to be inversed to seal the top, and the interlocking process is the same as former layers, as shown in Figure 3c. During the interlocking process, adhesive or welding flux should be smeared in the interlocking slots. After being interlocked, waiting for the curing of the adhesive or the finish of braze-welding process, the component parts interlocked will become a complete 3D auxetic structure. Compare the original 3D re-entrant structure (Figure 3a) and the structure obtained from the interlocking (Figure 3d), it can be seen that the small blocks added will not significantly influence the shape and properties of the auxetic structure.

It should be mentioned that, the number of the unit cell in any of the three directions is not restricted. Theoretically, arbitrary size 3D auxetic structure can be manufactured using this interlocking process. Additionally, as mentioned in the reference each component part can be individually qualified [37]. And the whole 3D auxetic structure will avoid the defects caused by additive manufacturing method.

As mentioned before, the novel method is suitable for any kind of material that can be adhesively bonded or welded. The structures using both polymer and metal have been successfully manufactured, as shown in Figure 5. The polymer structure was manufactured by interlocking and adhesively bonding the four types of polymer parts. And the following content focus on the study of the metal structures. 


\section{Experiments}

The specimens for mechanical testing were built with the interlocking assembly method introduced above using commercially available 304 stainless steel (304ss). Firstly, the four types of 304ss component parts were manufactured by wire-electrode cutting. The machining accuracy of the wire-electrode cutting machine used in present study is $\pm 0.01 \mathrm{~mm}$. The accuracies of the lateral dimensions are determined by the cutting process and not influenced by the interlocking process, and only the height may be slightly influenced by the interlocking errors. Before interlocking the component parts were cleaned and degreased. And then they were interlocked with welding flux (mixture of polymer based binder and a Nickel based metallic braze powder BNi2) smeared in the interlocking slots. After that, the interlocked structures were placed in a vacuum furnace for braze-welding. At a pressure of $\sim 10^{-4}$ torr, the specimens were heated at $10{ }^{\circ} \mathrm{C} / \mathrm{min}$ to $900{ }^{\circ} \mathrm{C}$ and held for 1 hour to volatilize the binder material. The temperature was then increased to $1050{ }^{\circ} \mathrm{C}$ and held for 1 hour to allow the braze material to liquefy. The specimens were then furnace cooled to ambient temperature leaving the specimens in an annealed state. The braze-welding process is similar with that used by Biagi [36]. After the braze-welding process, complete 3D auxetic structures were obtained as shown in Figure $\mathbf{5 b}$ from which it can be seen that both vertical struts and oblique struts have smooth surfaces and high quality.

The specimens with $5 \times 5 \times 3$ unit cell were chosen. The parameter values for design configuration are as follows: $H=16.00 \mathrm{~mm}, L=8.00 \mathrm{~mm}, t=1.79 \mathrm{~mm}$. The re-entrant angle is changing from $30^{\circ}$ to $70^{\circ}$ with an interval of $10^{\circ}$ to study the influence of the re-entrant angle on the mechanical properties of the structure, and for each degree three specimens were built.

Compressive testing was performed using Materials Testing Machines Instron 5569 at a crosshead speed of $0.1 \mathrm{~mm} / \mathrm{min}$. Similar to the method adopted by Schwerdtfeger [17], strain along the force 
axis and normal to it were measured using laser extensometer (Model LE-05, EIR Ltd., Irwin, PA) in company with retroreflective laser tags. And the tests were limited in the scope of $\varepsilon_{y} \leq 0.001$ to ensure that the specimens are in their elastic region. The schematic diagram of the compressive testing setup is shown in Figure 6a. To measure the vertical deformation of the specimen, two retroreflective laser tags were attached to the platens, one on the upper platen and the other on the lower platen, as shown in the schematic diagram, and then the laser extensometer can trace the relative displacement between the two tags which reflects the relative displacement between the two platens and the vertical deformation of the specimen. Similarly, two retroreflective laser tags were attached to the center of the outmost vertical struts of the center layer to measure the lateral deformation of the specimen, as shown in the schematic diagram.

\section{Numerical simulations}

In order to evaluate and probe the mechanical properties of the structures under compression, numerical simulations were performed. The finite element analysis software ABAQUS/Standard was employed for the simulations. The numerical model for compression tests is depicted in Figure 6b. The structures were meshed with structured 8-node linear brick incompatible C3D8I elements. Mesh convergence study shows that average element size of $0.67 \mathrm{~mm}$ gives accurate results within reasonable calculation time. The number of elements is different for different specimen, take the model of the specimen with $\theta=45^{\circ}, t=1.79 \mathrm{~mm}, H=2 L=16 \mathrm{~mm}$ for example, the finite element mesh consisted of 212103 elements comprising a total of 343008 nodes. The platens were meshed with rigid elements. Surface-to-surface contacts were defined between the platens and the specimen. The contact interaction property of the surface-to-surface contacts is defined in two aspects, tangential behavior and normal behavior. Friction coefficient of 0.1 is defined for the tangential behavior, and "Hard" contact is defined for the normal behavior. The lower platen was 
fixed and a downward displacement load about one percent of the specimens height was exerted on the reference point of the upper platen, as the boundary condition settings and load conditions of the simulations. The relative displacement of the nodes in the center of the outmost opposite two vertical struts in the center layer of the structure was used to calculate the transverse strain of the structure, as shown in Figure 6b.

Tension tests were performed on tensile specimens of dog-bone geometry cut from the 304 stainless steel sheet to obtain the parent material constitutive response which is needed as an input for the finite element simulations. In order to get the material properties that would closely resemble those of the 3D re-entrant auxetic structure, the specimens for tension tests were placed in the vacuum furnace with the assembled structures to suffer the same thermal cycle. The material properties obtained are as follows: the Young's modulus $E_{s}=196 \mathrm{GPa}$, Poisson's ratio $v_{s}=0.295$ and yield stress $\sigma_{s}=185 \mathrm{MPa}(0.2 \%$ offset $)$.

Utilizing the numerical model mentioned above, the compression proprieties of the specimens with variation re-entrant angles were firstly simulated. The re-entrant angle variation from $30^{\circ}$ to $70^{\circ}$ with an interval of $2.5^{\circ}$ was considered in the numerical simulation. And then the simulated results were compared with the experimental results. Based on the experimentally verified numerical model, the effects of the strut thickness $t$ and $\alpha$ on the mechanical properties were analyzed. To study the effects of the strut thickness $t$, the re-entrant angle $\theta$ and $\alpha$ were fixed at $\theta=45^{\circ}$ and $\alpha=2$. And $t$ must satisfy the geometric compatibility constrains in inequation (2) and inequation (3) which require $t<4 \sqrt{2} \mathrm{~mm}$. So models with $t$ changing from $0.4 \mathrm{~mm}$ to $5 \mathrm{~mm}$ with an interval of $0.2 \mathrm{~mm}$ were simulated. To study the effects of $\alpha$, the re-entrant angle $\theta$ and the strut thickness $t$ were fixed at $\theta=45^{\circ}$ and $t=1.79 \mathrm{~mm}$. And $\alpha$ must satisfy the geometric compatibility constrains in inequation (2) which leave limited range for the increase of the oblique 
strut length $L$, so $L$ was fixed, and $\alpha$ was changed by changing $H$. With $L=8.00 \mathrm{~mm}$ the limitation for $H$ is $H>9.79 \times \sqrt{2}-1.79 \mathrm{~mm}$; and for $\alpha$, it must be larger than $(9.79 \times \sqrt{2}-1.79) / 8$. So models with $\alpha$ changing from 1.6 to 3.2 with an interval of 0.2 were simulated.

\section{Results and discussion}

$X_{1}$ and $X_{2}$ are not distinguished during results analyses, as the structure is symmetric in the lateral direction. For both experimental and numerical, the Young's moduli of the specimens were found directly from the slope of stress-strain curves, and the Poisson's ratios of the specimens were obtained from the slope of $\varepsilon_{x}-\varepsilon_{y}$ curves. The stress-strain curves and the $\varepsilon_{x}-\varepsilon_{y}$ curves of five specimens (one specimen selected at each angle) have been shown in Figure 7. From the curves, the Young's moduli and Poisson's ratio $v_{y x}$ of the specimens can be obtained.

Figure 8 shows the numerical results of the Mises stress contour plot and X-direction displacement contour plot for structure with $\theta=45^{\circ}, t=1.79 \mathrm{~mm}$ and $L=H / 2=8.00 \mathrm{~mm}$. One distinctive feature of the stress distribution is that high stress areas mainly concentrate on the oblique struts. The high stress regions distribute on both sides of the oblique struts axles and have certain distance from the joints of the oblique struts and vertical struts, which implies the bending deformation mechanism of the oblique struts, as shown in the ellipse in Figure 8a. According to former studies [27], the bending of the oblique strut is the main deformation mechanism of the structure and the main mechanism that generate the auxetic nature of the structure. As shown in Figure $\mathbf{8 b}$, in the lateral direction the outside materials will move inward under compression which verifies the auxetic nature of the structure. The bending outward of the outmost vertical struts in the center due to the edge effect can also be seen in Figure 8b. The bending outward of the outmost vertical struts 
partly offset the lateral contraction of the structure under compression, which result in less auxetic of the structure and relatively conservative Poisson's ratio.

\subsection{The effect of re-entrant angle}

Based on the methods outlined above, the Young's modulus and Poisson's ratio for all specimens can be obtained. The experimental results were compared with the numerical results, as shown in Figure 9. As can be seen in Figure 9a, the Young's modulus of the specimens will monotonically decrease with the increase of re-entrant angle, and the experimental results match well with the numerical results though slightly lower. The main reason behind this difference may be the weakness of the structure near the welding point. The property of welding material is not as strong as the parent metal and there may be defects in the welding point. These two reasons combined will weaken the structure near the welding point.

The weakness near the joints is mainly taken as defect to the structure; however in certain case the weakness was deliberately designed to make the structures more auxetic $[17,38]$. Furthermore, from the stress contour plot as shown in Figure 8a, it can be seen that, the area near the joint is not the high stress region which implies that the weakness near the joints may not reduce the strength of the structure. In all, the weakness near the joints will not severely influence the strength of the structure, while it can make the auxetic nature of the structure more pronounced.

From Figure 9b, it can be observed that there exists an extreme value for Poisson's ratio with the re-entrant angle around $45^{\circ}$. The absolute value of the Poisson's ratio $v_{y x}$ will decrease (less auxetic) with the increase of the re-entrant angle when the angle is larger than the extreme point, conversely, the absolute value of the Poisson's ratio $v_{y x}$ will also decrease with the decrease of the re-entrant angle when the angle is smaller than the extreme point. This phenomenon can be interpreted as follows: when the angle is larger than the extreme point, with the increase of the 
angle the structure will become less re-entrant, though the re-entrant character is the source of auxetic, so with the increase of the angle the structure will become less auxetic. When the angle is smaller than the extreme point, with the decrease of the angle the oblique struts will more inclined to the vertical strut and will bear more axial load and less bending load, though the main mechanism causing the auxetic character of the structure is the flexure of the oblique struts, so with the decrease of the angle the structure will become less auxetic. Besides, because of the overlapping of the vertical struts and oblique struts due to the thickness of the struts, the effective length of the oblique struts for bending is shorter than the design values [18]. It is not easy to accurately estimate the reduction of the effective length $\Delta L$. It can be calculated by extending the vertical strut line, as line-1 shown in Figure 10, and the reduction $\Delta L_{1}$ could be estimated as

$$
\Delta L_{1}=\frac{t}{\sin \theta}
$$

Or it can be simply estimated by constructing a perpendicular line to the oblique strut line passing through the intersection point of the oblique strut edge line and the vertical strut edge line, as line-2 shown in Figure 10, and the reduction $\Delta L_{2}$ could be estimated as

$$
\Delta L_{2}=\frac{(1+\cos \theta) t}{\sin \theta}
$$

The real the reduction of the effective length $\Delta L$ may fall in between $\Delta L_{1}$ and $\Delta L_{2}$

$$
\frac{t}{\sin \theta}<\Delta L<\frac{(1+\cos \theta) t}{\sin \theta}
$$

With the decrease of the re-entrant angle the vertical struts and oblique struts will get more overlapped, and also from the reduction estimation it can be seen that the effective length of the oblique struts for bending will get shorter. Finally the decrease of the angle will make the structure less auxetic. 
The parabolic relationship between the Poisson's ratio and the re-entrant angle found in the present study is contrary to the monotonic relationship predicted by former theories [39-41]. The reason behind this may be that the former theories are based on beam theories which require the strut of the structure to be slender ( $t / L$ is small). However, in the present study, the struts of the specimens are relatively stubby. So the related theories are inapplicable to the analysis of specimens in the present study.

From Figure 9b it can also be seen that the experimental results of the absolute value of the Poisson's ratio $v_{y x}$ are larger (more auxetic) than the numerical results. The main reason behind this difference may also be the weakness of the structure near the welding point. The weakness will work as hinge-like elements which will make the structure more auxetic, and this phenomenon is in accordance with the optimization result by Schwerdtfeger [17].

\subsection{The effect of strut thickness}

The numerical results of the relationship between the structure's normalized compression Young's modulus as well as Poisson's ratio $v_{y x}$ and the strut thickness coupled with the relationship between the structures' relative density and the strut thickness have been plotted in Figure 11a and

Figure 11b respectively. As shown in the figure and Equation (4), the structure's relative density increase with the increase of strut thickness. As pointed out by Gibson and Ashby [39], all mechanical properties including the compression Young's modulus scale with the relative density of the structure for cellular structures. As expected, the normalized compression Young's modulus of the structures increases with the increase of the strut thickness.

As shown in Figure 11b, the structure's Poisson's ratio $v_{y x}$ is strongly depend on the strut thickness. The increase of strut thickness will lead to a decrease of the absolute value of the Poisson's ratio until at about $t=2.8 \mathrm{~mm}$ the auxetic nature of the structure will disappear 
completely. With the continuous thickening of the struts, the Poisson's ratio $v_{y x}$ of the structure will gradually approach the Poisson's ratio of the parent material.

The effect of strut thickness on the shape of the structure can be seen in Figure 12. The gradual change of the structure's deformation from contraction to expansion in lateral direction under uniaxial compression can also be seen in Figure 12. Due to the successive space shrinking, the folding up mechanism responsible for the auxetic nature of the structure will gradually shut down with the thickening of the strut. With the thickening of the strut, more area will get overlapped at the connection of the vertical strut and the oblique strut, the effective length for the oblique strut will get shorter, which gradually lead the change of the dominated deformation from bending to a more mixed deformation mode comprising of bending, shearing and stretching. This phenomenon is similar to the structure studied by Schwerdtfeger [27]. However, stretching of the struts will lead to a positive Poisson's ratio for re-entrant structure [42]. The increase of the shearing/stretching contribution to the deformation leads to a decrease of the absolute value for the negative Poisson's ratios [27].

\subsection{The effect of $\alpha$}

The effect of $\alpha$ on the normalized effective modulus $E_{y} / E_{s}$ is shown in Figure 13a. It is clear that with the increase of $\alpha$, the normalized effective modulus $E_{y} / E_{s}$ of the structure will increase monotonously. This conclusion is intuitive since the increase of the total dimension in $Y$ will result in a decrease of the strain values, and in turn larger modulus. It can also be seen that the slope of the $\alpha-E_{y} / E_{x}$ curve, which means the increase rate of the normalized effective modulus $E_{y} / E_{s}$, decreases monotonously, at high $\alpha$ value the effect of the increase of $\alpha$ on the normalized effective modulus $E_{y} / E_{s}$, will gradually weaken. The increase of $\alpha$ can be considered to the elongations of the vertical struts, as shown in Figure 13c. In view of "series 
model", it can be predicted that the value of the normalized effective modulus $E_{y} / E_{s}$ of the structure will not exceed that of the newly added portion. The value of the normalized effective modulus of the newly added portion can be calculated by the ratio of vertical struts cross section area in one unit cell to the cross section area of the unit cell which yields $2 t^{2} /(2 L \cdot \sin \theta)^{2}=1 / 2[t /(L \cdot \sin \theta)]^{2}$, and for the given parameters, it won't exceed 0.05 .

The relationship between the structure's Poisson's ratio $v_{y x}$ and $\alpha$ has been plotted as shown in Figure 13b. The parabolic relationship between the Poisson's ratio $v_{y x}$ and $\alpha$ can be seen. At low $\alpha$ value, the absolute value of the Poisson's ratio $v_{y x}$ increase with the increase of $\alpha$, this phenomenon is intuitive since the increase of $H$ will result in the increase of the structure's total dimension in $Y$ which will result in a decrease of the strain values, and with the lateral strain unchanged, the structure will become more auxetic. However in the large $\alpha$ value range, with the elongation of the vertical strut, the bending outward of the outmost struts becomes more dominant, which can be seen from Figure 13d by comparing the lateral displacement contours of the center layer of the specimens with $\alpha=1.6$ and $\alpha=2.4$. The bending outward of the outmost struts will offset the effect of $\alpha$ increasing or even make the structure less auxetic, as shown in Figure 13b. By comparing the effects of the re-entrant angle $\theta$, the strut thickness $t$ and $\alpha$, it could be clearly observed that the re-entrant angle $\theta$ and the strut thickness $t$ have more significant effect on the effective modulus in $Y$ compared with $\alpha$.

\section{Conclusions}

The present work proposed an interlocking assembly method that can be used to manufacture 3D auxetic cellular structure, and this manufacturing method has great advantages compared with additive manufacturing method. A series of specimens have been successfully built using this method. The auxetic nature of the structures has been demonstrated by compression testing. And the 
dependences of Poisson's ratio and Young's modulus of the structure on the reentrant angle were found numerically and experimentally. Based on the experimentally verified numerical model, the effects of the strut thickness and the ratio of the vertical strut length to oblique strut length on the Poisson's ratio and the Young's modulus were simulated in turn. The experimental and numerical results have shown the significant dependence of the mechanical properties of the 3D auxetic structure subjected to uniaxial loading on the geometric parameters.

It should be noted the assembly concept can also be introduced to other 3D periodic auxetic cellular structures which is under studying.

\section{Acknowledgements}

The present work is supported by the National Science Foundation of China under Grant Nos. 11172080 and 11222216.

\section{References}

[1] Nur A, Simmons G. The effect of saturation on velocity in low porosity rocks. Earth and Planetary Science Letters. 1969;7:183-93.

[2] Veronda D, Westmann R. Mechanical characterization of skin - finite deformations. Journal of biomechanics. 1970;3:111-24.

[3] Gunton D, Saunders G. The Young's modulus and Poisson's ratio of arsenic, antimony and bismuth. Journal of Materials Science. 1972;7:1061-8.

[4] Li Y. The anisotropic behavior of Poisson's ratio, Young's modulus, and shear modulus in hexagonal materials. physica status solidi (a). 1976;38:171-5.

[5] Williams J, Lewis J. Properties and an anisotropic model of cancellous bone from the proximal tibial epiphysis. Journal of biomechanical engineering. 1982;104:50-6.

[6] Lakes R. Foam structures with a negative Poisson's ratio. Science. 1987;235:1038-40. 
[7] Evans KE, Alderson A. Auxetic materials: functional materials and structures from lateral thinking! Advanced materials. 2000;12:617-28.

[8] Lakes R, Elms K. Indentability of conventional and negative Poisson's ratio foams. Journal of Composite Materials. 1993;27:1193-202.

[9] Alderson K, Fitzgerald A, Evans K. The strain dependent indentation resilience of auxetic microporous polyethylene. Journal of Materials Science. 2000;35:4039-47.

[10] Choi J, Lakes R. Fracture toughness of re-entrant foam materials with a negative Poisson's ratio: experiment and analysis. International Journal of Fracture. 1996;80:73-83.

[11] Scarpa F, Ciffo L, Yates J. Dynamic properties of high structural integrity auxetic open cell foam. Smart materials and structures. 2004;13:49.

[12] Alderson A, Rasburn J, Evans K. Mass transport properties of auxetic (negative Poisson's ratio) foams. physica status solidi (b). 2007;244:817-27.

[13] Grima J, Jackson R, Alderson A, Evans K. Do zeolites have negative Poisson's ratios? Advanced materials. 2000;12:1912-8.

[14] Evans K. The design of doubly curved sandwich panels with honeycomb cores. Composite Structures. 1991; 17:95-111.

[15] Liu Y, Hu H. A review on auxetic structures and polymeric materials. Sci Res Essays. 2010;5:1052-63.

[16] Lim T-C. Auxetic Materials and Structures: Springer, 2014.

[17] Schwerdtfeger J, Wein F, Leugering G, Singer RF, Korner C, Stingl M, et al. Design of auxetic structures via mathematical optimization. Adv Mater. 2011;23:2650-4.

[18] Yang L. Structural Design, Optimization and Application of 3D Re-entrant Auxetic Structures: North Carolina State University, 2012. 
[19] Yang L, Harrysson O, West H, Cormier D. Compressive properties of Ti-6Al-4V auxetic mesh structures made by electron beam melting. Acta Materialia. 2012;60:3370-9.

[20] Andreassen E, Lazarov BS, Sigmund O. Design of manufacturable 3D extremal elastic microstructure. Mechanics of Materials. 2014;69:1-10.

[21] Buckmann T, Stenger N, Kadic M, Kaschke J, Frolich A, Kennerknecht T, et al. Tailored 3D mechanical metamaterials made by dip-in direct-laser-writing optical lithography. Adv Mater. 2012;24:2710-4.

[22] Critchley R, Corni I, Wharton JA, Walsh FC, Wood RJ, Stokes KR. The Preparation of Auxetic Foams by Three - Dimensional Printing and Their Characteristics. Advanced Engineering Materials. 2013;15:980-5.

[23] Babaee S, Shim J, Weaver JC, Chen ER, Patel N, Bertoldi K. 3D soft metamaterials with negative Poisson's ratio. Advanced materials. 2013;25:5044-9.

[24] Shokri Rad M, Prawoto Y, Ahmad Z. Analytical solution and finite element approach to the 3D re-entrant structures of auxetic materials. Mechanics of Materials. 2014;74:76-87.

[25] Schwerdtfeger J, Heinl P, Singer RF, Körner C. Auxetic cellular structures through selective electron-beam melting. physica status solidi (b). 2010;247:269-72.

[26] Rad MS, Ahmad Z, Alias A. Computational approach in formulating mechanical characteristics of 3D star honeycomb auxetic structure.

[27] Schwerdtfeger J, Schury F, Stingl M, Wein F, Singer RF, Körner C. Mechanical characterisation of a periodic auxetic structure produced by SEBM. physica status solidi (b). 2012;249:1347-52.

[28] Ravirala N, Alderson A, Alderson K. Interlocking hexagons model for auxetic behaviour. Journal of Materials Science. 2007;42:7433-45. 
[29] Robert F. An isotropic three-dimensional structure with Poisson's ratio--1. Journal of Elasticity. 1985;15:427-30.

[30] Evans K, Nkansah M, Hutchinson I. Auxetic foams: modelling negative Poisson's ratios. Acta metallurgica et materialia. 1994;42:1289-94.

[31] Dos Reis F, Ganghoffer J. Equivalent mechanical properties of auxetic lattices from discrete homogenization. Computational Materials Science. 2012;51:314-21.

[32] El Nady K, Ganghoffer J. Computation of the effective mechanical response of biological networks accounting for large configuration changes. Journal of the mechanical behavior of biomedical materials. 2015 .

[33] Grima JN, Caruana-Gauci R, Attard D, Gatt R. Three-dimensional cellular structures with negative Poisson's ratio and negative compressibility properties. Proceedings of the Royal Society of London A: Mathematical, Physical and Engineering Sciences: The Royal Society; 2012. p. 3121-38.

[34] Grima JN, Caruana-Gauci R, Wojciechowski KW, Evans KE. Smart hexagonal truss systems exhibiting negative compressibility through constrained angle stretching. Smart materials and structures. 2013;22:084015.

[35] Scarpa F, Tomlinson G. Theoretical characteristics of the vibration of sandwich plates with in-plane negative Poisson's ratio values. Journal of sound and vibration. 2000;230:45-67.

[36] Biagi R, Bart-Smith H. Imperfection sensitivity of pyramidal core sandwich structures. International Journal of Solids and Structures. 2007;44:4690-706.

[37] Cheung KC, Gershenfeld N. Reversibly Assembled Cellular Composite Materials. Science. 2013;341:1219-21.

[38] Wang K, Chang Y-H, Chen Y, Zhang C, Wang B. Designable dual-material auxetic 
metamaterials using three-dimensional printing. Materials \& Design. 2015;67:159-64.

[39] Gibson LJ, Ashby MF. Cellular solids: structure and properties: Cambridge university press, 1997.

[40] Wan H, Ohtaki H, Kotosaka S, Hu G. A study of negative Poisson's ratios in auxetic honeycombs based on a large deflection model. European Journal of Mechanics-A/Solids. 2004;23:95-106.

[41] Yang L, Harrysson O, West H, Cormier D. Mechanical properties of 3D re-entrant honeycomb auxetic structures realized via additive manufacturing. International Journal of Solids and Structures. 2015.

[42] Masters I, Evans K. Models for the elastic deformation of honeycombs. Composite Structures. 1996;35:403-22. 


\section{Figure Captions:}

Figure 1 (a) Actual surface of a strut made by EBM and measurement of strut dimensions[18] (b) the surface of a strut manufactured by wire-electrode cutting (c) cross section of strut made by EBM [18] (d) illustration of the stair stepping effect [27].

Figure 2 (a) 2D re-entrant auxetic structure, (b) 3D re-entrant auxetic structure, and umbrella shaped elements, (c) the unit cell of the 3D auxetic structure, (d) In-plane parameters needed for describing 2D re-entrant auxetic structure, (e) More parameters needed for describing 3D re-entrant auxetic structure, the depth of the vertical strut $t_{3}$ and oblique strut $t_{4}$.

Figure 3 (a) The 3D re-entrant auxetic structure; (b) the four types of 2D re-entrant component parts decomposed from the 3D re-entrant auxetic structure; (c) process of interlocking, the red arrow indicated the interlocking method of the component parts (small blocks in dark color added to the ends of the parts); (d) The 3D re-entrant auxetic structure being interlocking assembled from modified component parts.

Figure 4 Illustration of the manufacture of Part-1 and Part-2 by cutting a panel sheet.

Figure 5 (a) Polymer 3D auxetic specimen manufactured using the interlocking assembly method;

(b) 304ss 3D reentrant auxetic structures manufactured using the interlocking assembly method.

Figure 6 (a) Schematic diagram of the compressive testing setup; (b) the numerical model for the compression test.

Figure 7 (a) Stress-strain curves of the specimens under compression; (b) $\varepsilon_{x}$ versus $\varepsilon_{y}$ curves of the specimens under compression.

Figure 8 (a) Mises stress contour plot at $\varepsilon_{y}=0.001$ for structure with $\theta=45^{\circ}, t=1.79 \mathrm{~mm}$ and $L=H / 2=8.00 \mathrm{~mm}$, (b) X-direction displacement contour plot at $\varepsilon_{y}=0.001$ for structure with $\theta=45^{\circ}, \quad t=1.79 \mathrm{~mm}$ and $L=H / 2=8.00 \mathrm{~mm}$. 
Figure 9 (a) Young's modulus plotted against re-entrant angle both the experimental results and the numerical results; (b) Poisson's ratio $v_{y x}$ plotted against re-entrant angle both the experimental results and the numerical results; error bar stands for standard deviation (SD).

Figure 10 The reduction of the effective length of the oblique struts for bending, $L_{1}$ and $L_{2}$ denote the effective length estimated.

Figure 11 (a) The dependence of the structure's normalized compression Young's modulus on the relative density of the structure (b) the dependence of the structure's Poisson's ratio $v_{y x}$ on the relative density of the structure.

Figure 12 The lateral displacement of the structures with different strut thickness.

Figure 13 (a) The dependence of the structure's normalized compression Young's modulus on $\alpha$; (b) the dependence of the structure's Poisson's ratio $v_{y x}$ on $\alpha$; (c) the effect of the increase of $\alpha$ on the geometry of the structure (illustrate by the comparison of the unit cells with $\alpha=1.6$ and $\alpha=2.4$ ); (d) the lateral displacement contour of the center layer of the specimens with $\alpha=1.6$ and $\alpha=2.4$. 


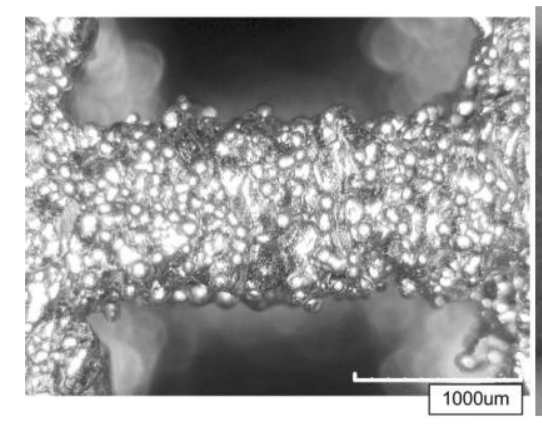

a)

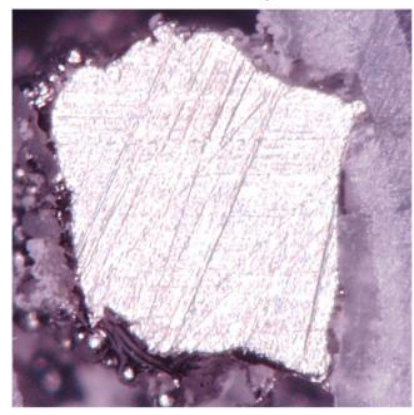

c)

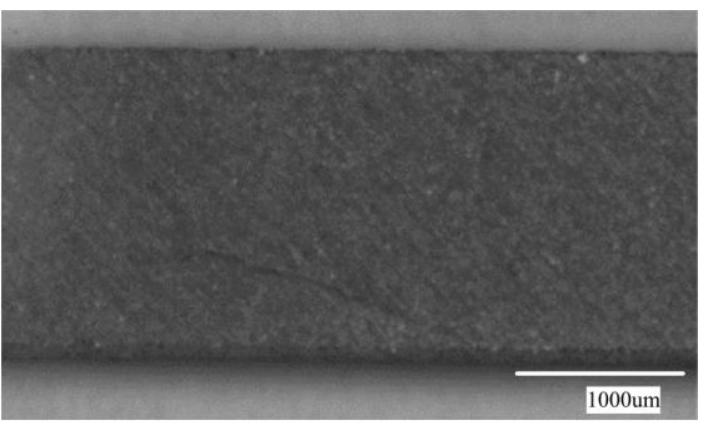

b)

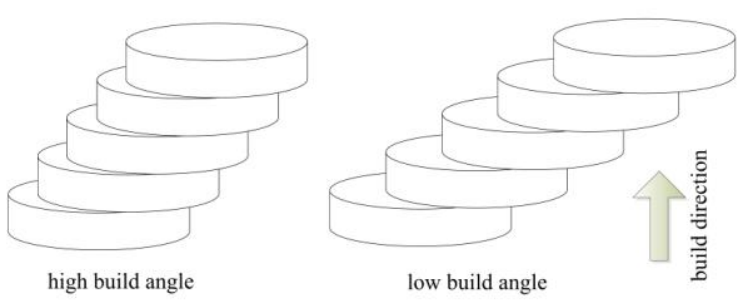

d)

Figure 1 (a) Actual surface of a strut made by EBM and measurement of strut dimensions[18] (b) the surface of a strut manufactured by wire-electrode cutting (c) cross section of strut made by EBM [18] (d) illustration of the stair stepping effect [27].
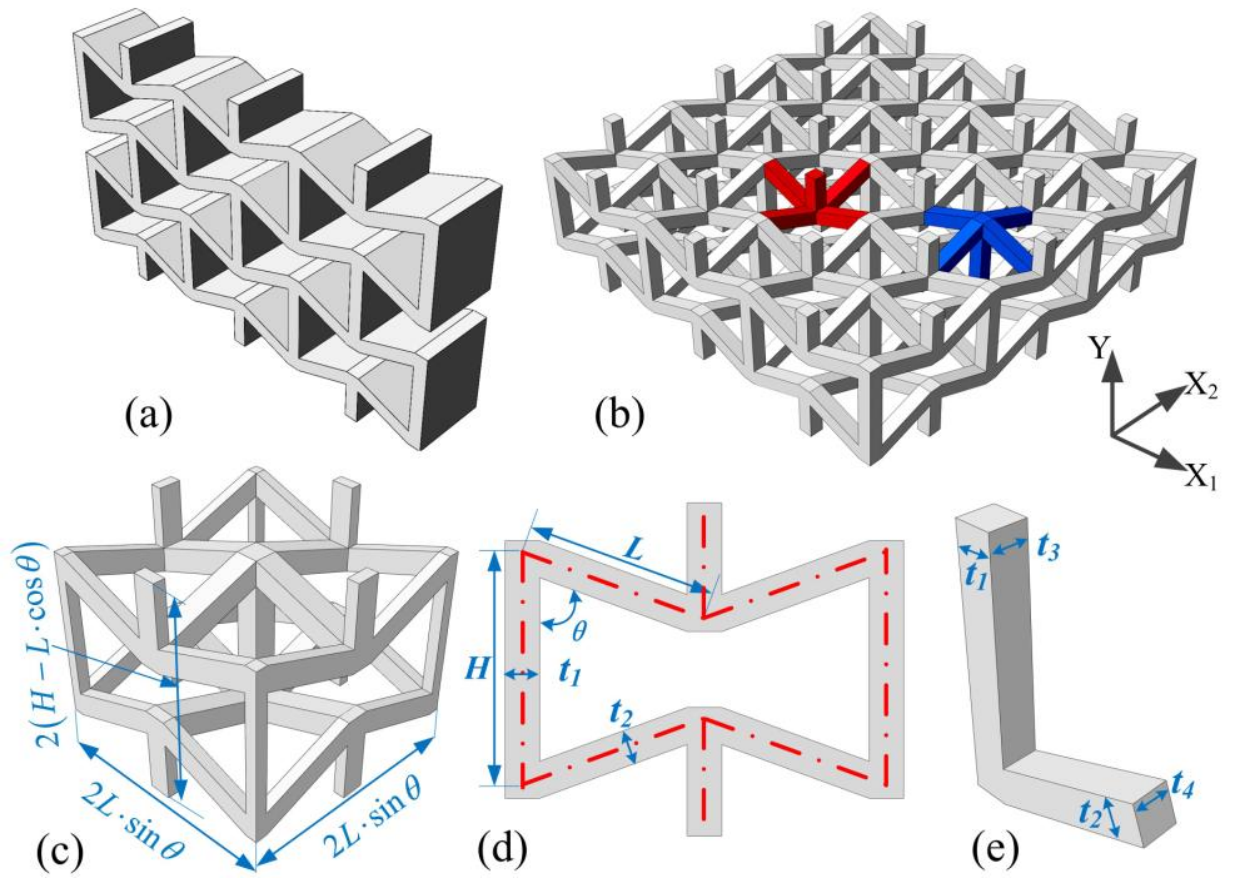

Figure 2 (a) 2D re-entrant auxetic structure, (b) 3D re-entrant auxetic structure, and umbrella shaped elements, (c) the unit cell of the 3D auxetic structure and its dimensions, (d) In-plane parameters needed for describing 2D re-entrant auxetic structure, (e) More parameters needed for describing 3D re-entrant auxetic structure, the depth of the vertical strut $t_{3}$ and oblique strut $t_{4}$. 


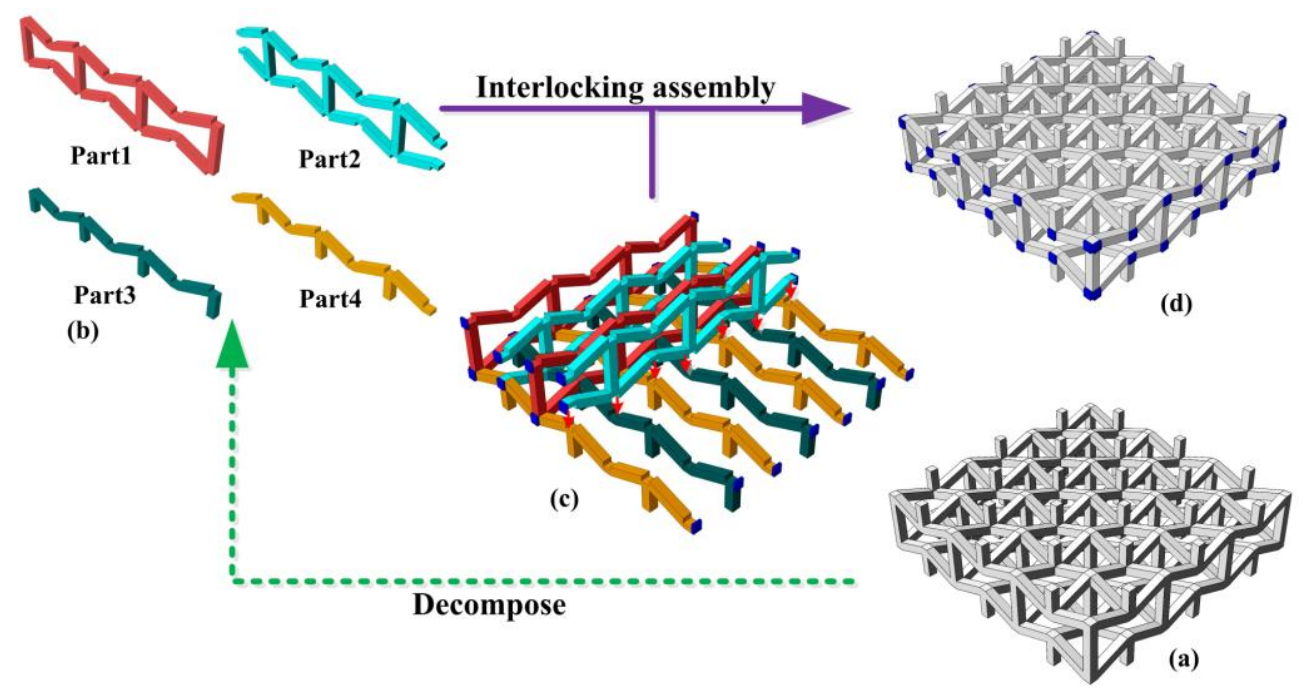

Figure 3 (a) The 3D re-entrant auxetic structure; (b) the four types of 2D re-entrant component parts decomposed from the 3D re-entrant auxetic structure; (c) process of interlocking, the red arrow indicated the interlocking method of the component parts (small blocks in dark color added to the ends of the parts); (d) The 3D re-entrant auxetic structure being interlocking assembled from modified component parts.

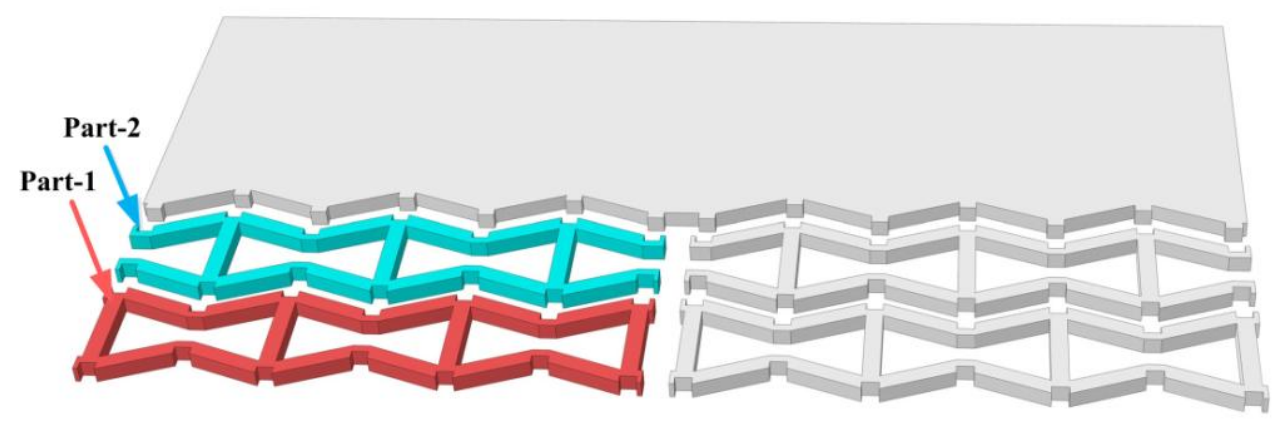

Figure 4 Illustration of the manufacture of Part-1 and Part-2 by cutting a panel sheet.

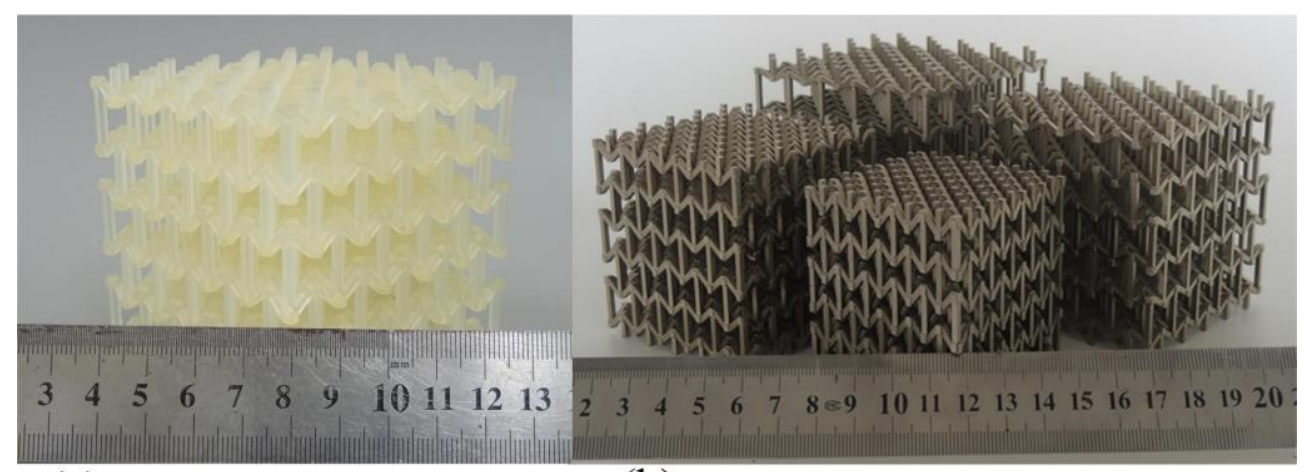

(a)

(b)

Figure 5 (a) Polymer 3D auxetic specimen manufactured using the interlocking assembly method; (b) 304ss 3D reentrant auxetic structures manufactured using the interlocking assembly method. 

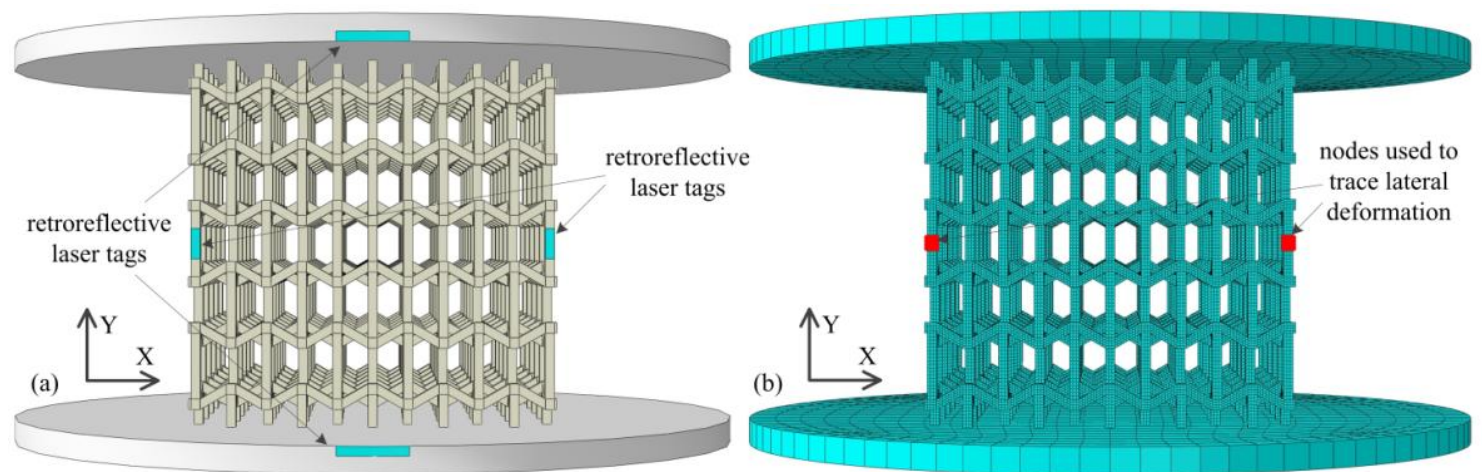

Figure 6 (a) Schematic diagram of the compressive testing setup; (b) the numerical model for the compression test.
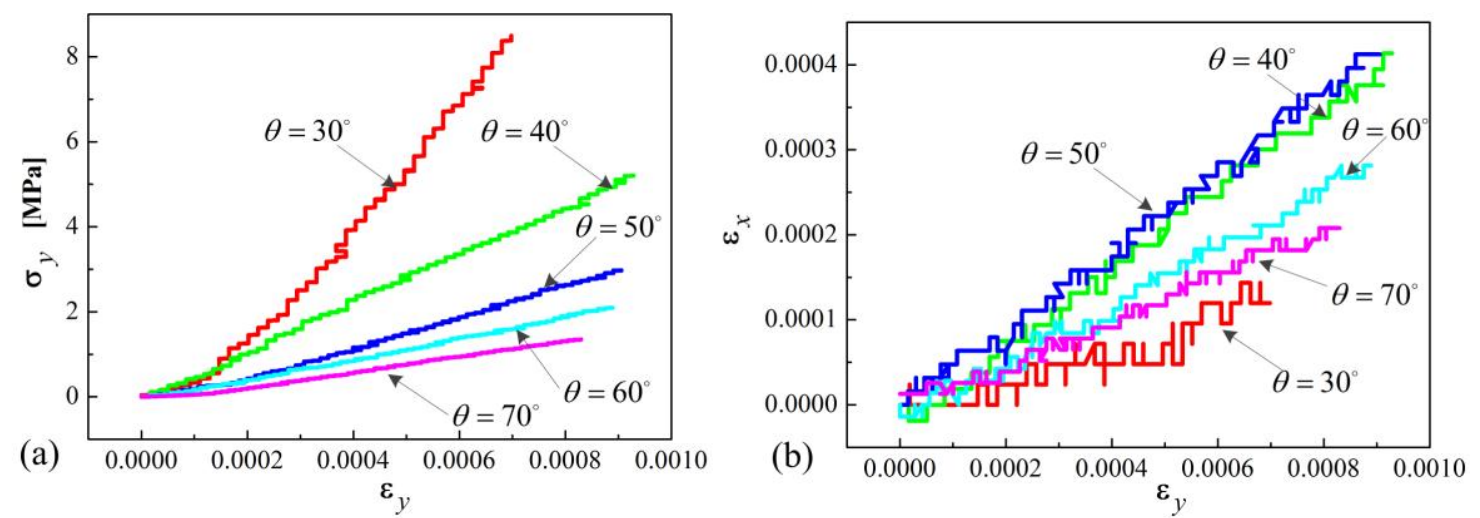

Figure 7 (a) Stress-strain curves of the specimens under compression; (b) $\varepsilon_{x}$ versus $\varepsilon_{y}$ curves of the specimens under compression.
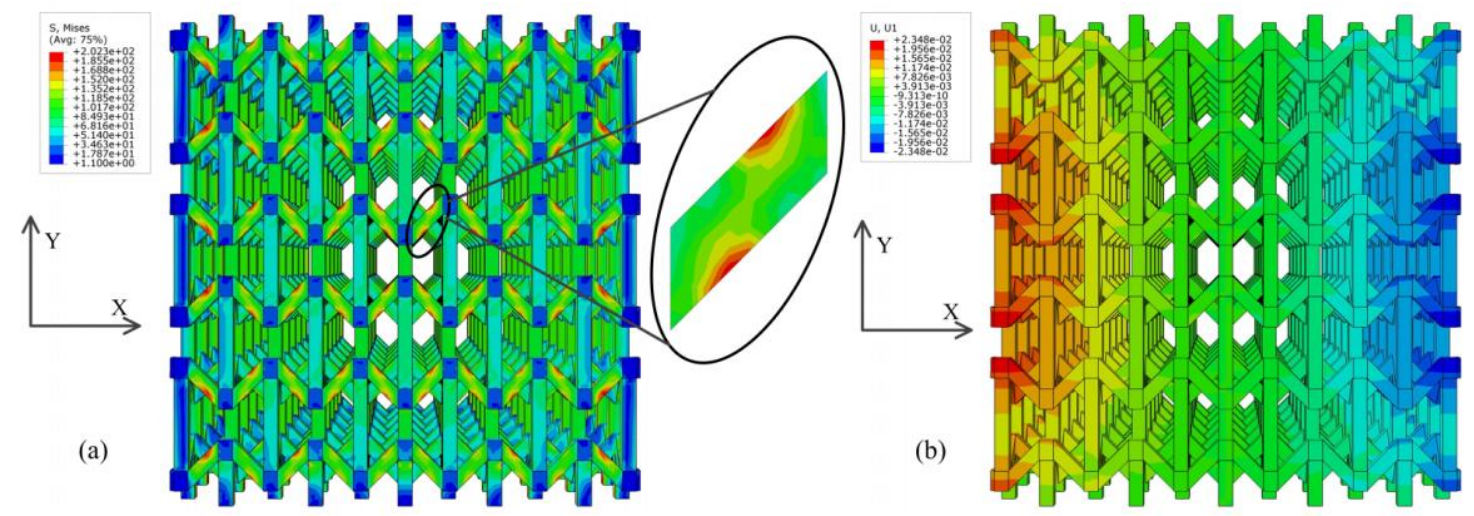

Figure 8 (a) Mises stress contour plot at $\varepsilon_{y}=0.001$ for structure with $\theta=45^{\circ}$, $t=1.79 \mathrm{~mm}$ and $L=H / 2=8.00 \mathrm{~mm}$, (b) X-direction displacement contour plot at $\varepsilon_{y}=0.001$ for structure with $\theta=45^{\circ}, t=1.79 \mathrm{~mm}$ and $L=H / 2=8.00 \mathrm{~mm}$. 


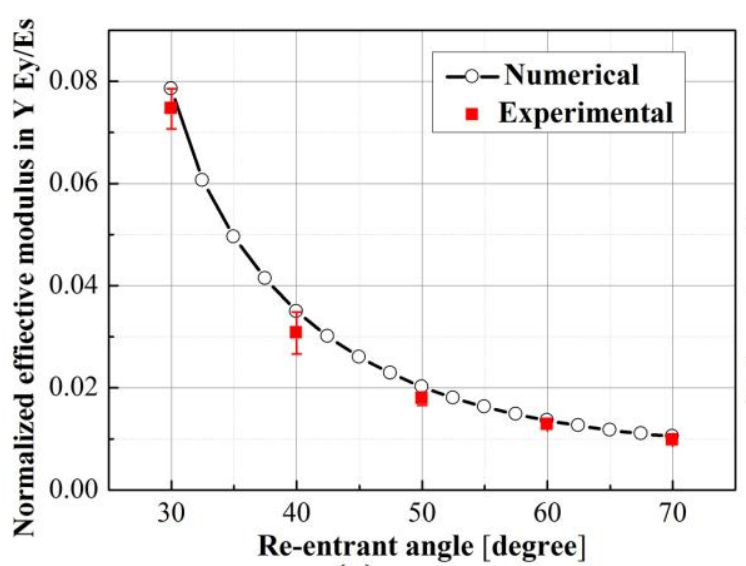

(a)

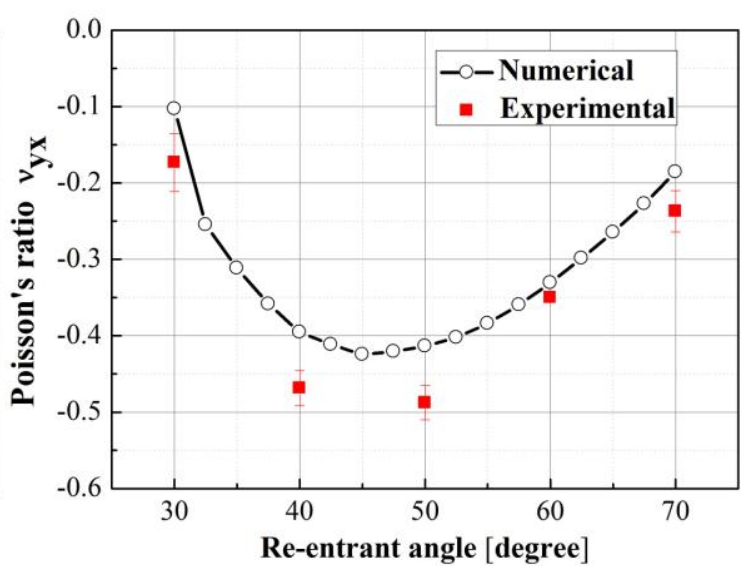

(b)

Figure 9 (a) Young's modulus plotted against re-entrant angle both the experimental results and the numerical results; (b) Poisson's ratio $v_{y x}$ plotted against re-entrant angle both the experimental results and the numerical results; error bar stands for standard deviation (SD)

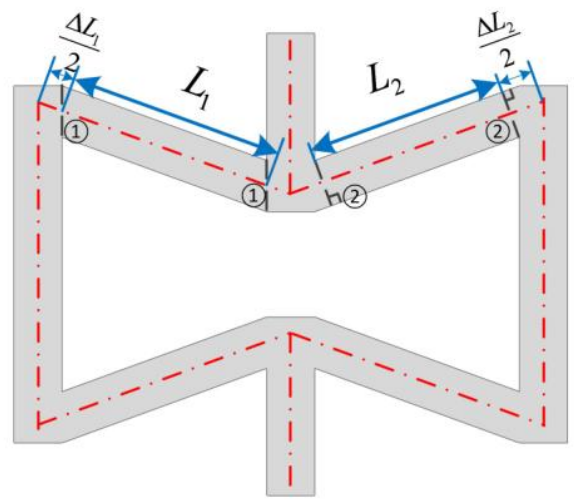

Figure 10 The reduction of the effective length of the oblique struts for bending, $L_{1}$ and $L_{2}$ denote the effective length estimated.

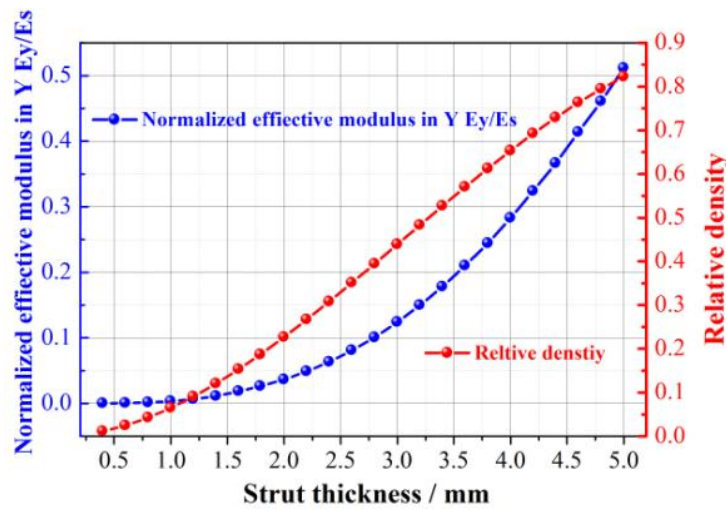

(a)

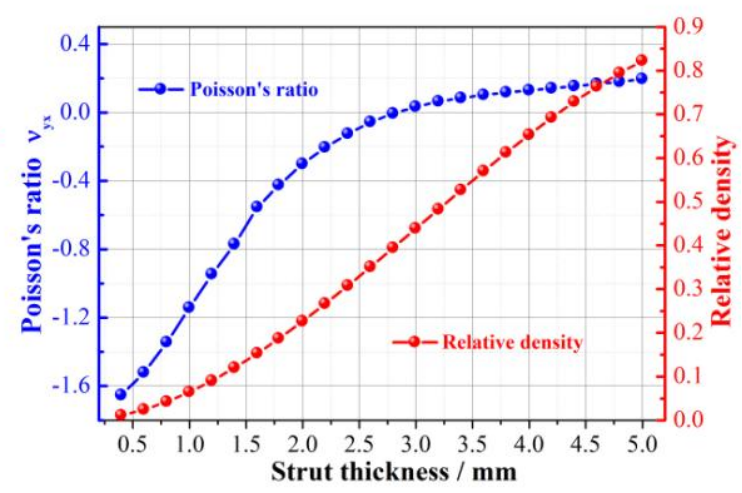

(b)

Figure 11 (a) The dependence of the structure's normalized compression Young's modulus on the relative density of the structure (b) the dependence of the structure's Poisson's ratio $v_{y x}$ on the relative density of the structure 


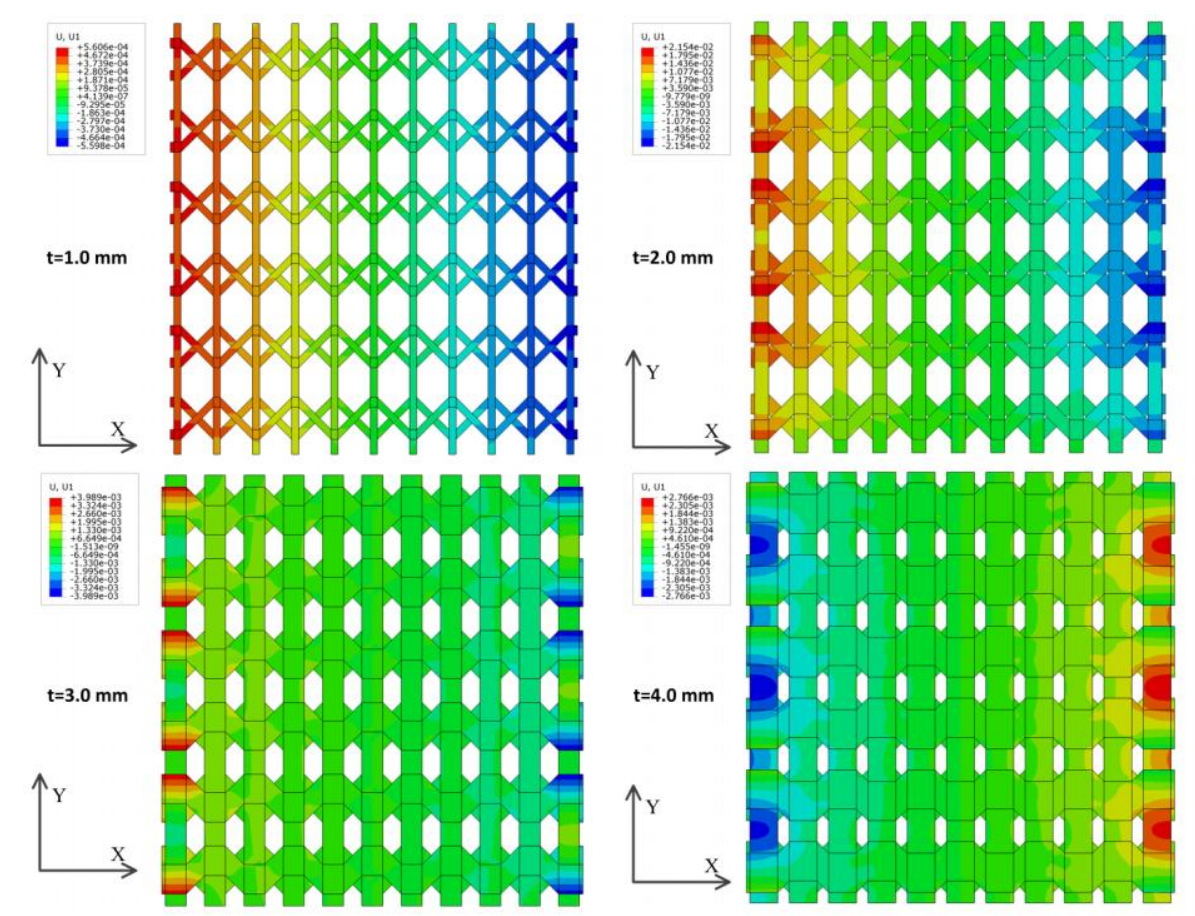

Figure 12 The lateral displacement of the structures with different strut thickness.
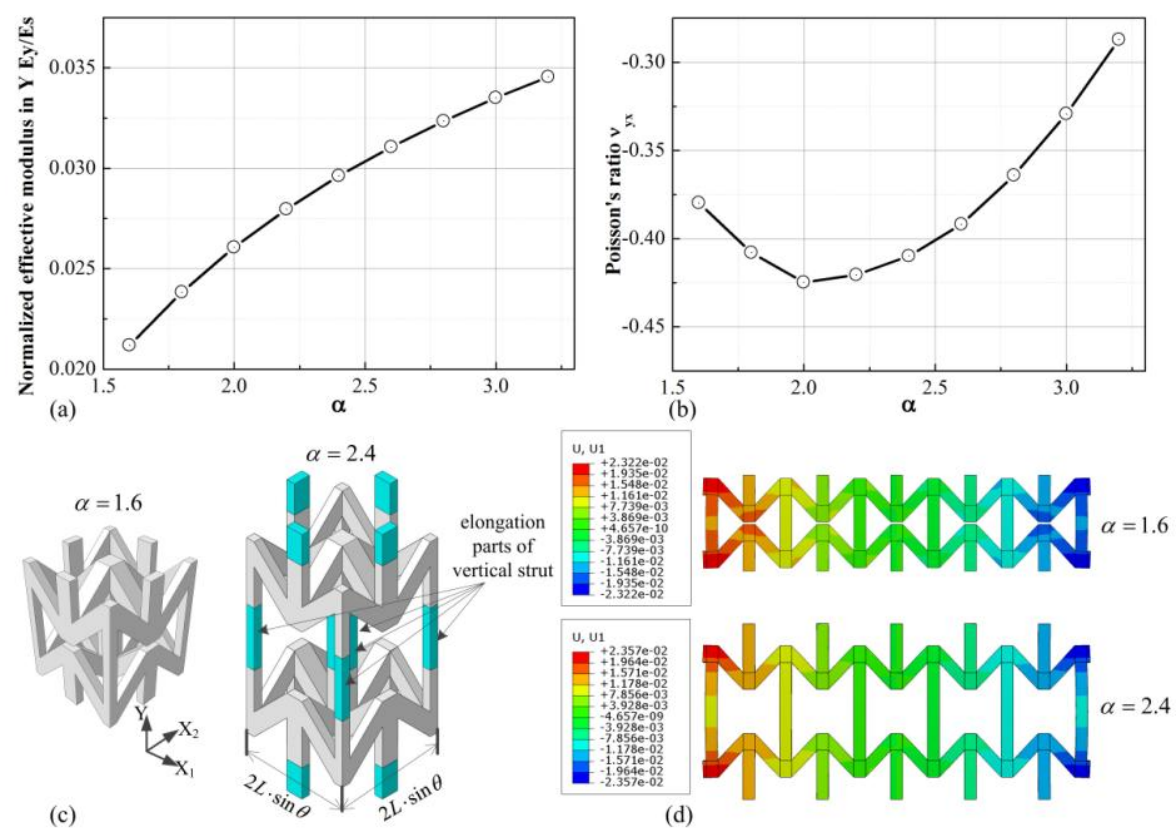

Figure 13 (a) The dependence of the structure's normalized compression Young's modulus on the ratio $\alpha$; (b) The dependence of the structure's Poisson's ratio $v_{y x}$ on the ratio $\alpha$; (c) The effect of the increase of ratio $\alpha$ on the geometry of the structure (illustrate by the comparison of the unit cells with $\alpha=1.6$ and $\alpha=2.4$ ); (d) The lateral displacement contour of the center layer of the specimens with $\alpha=1.6$ and $\alpha=2.4$. 

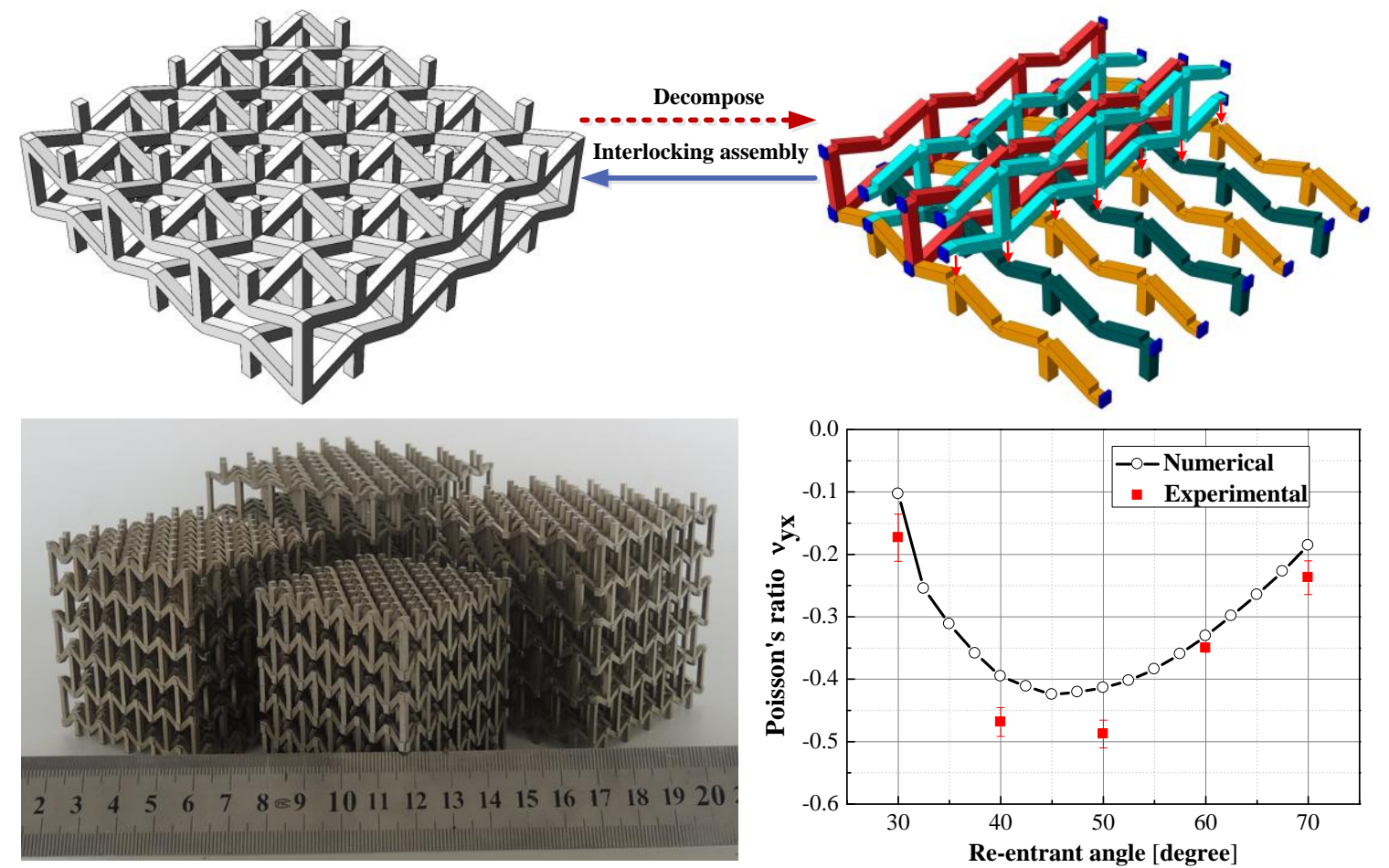